Yervant Terzian, Daniel Weedman, Edward Khachikian, eds.

\title{
Supernova Types, Star Formation and AGN
}

\author{
Massimo Turatto and Enrico Cappellaro \\ Osservatorio Astronomico di Padova, vicolo dell'Osservatorio 5, 35122 \\ Padova, Italy
}

Artashes R. Petrosian

\section{Byurakan Astrophysical Observatory, Aragatsotn Province, Armenia}

\begin{abstract}
New values of the frequencies of $\mathrm{SNe}$ are presented and discussed in relation to their use as SF indicators. The rate of core-collapse $\mathrm{SNe}$ is correlated to the colors and the FIR excesses of the parent galaxies in the sense that galaxies with blue colors and strong infrared excess have higher occurrence of type II and Ib/c SNe than other galaxies. This is in agreement with the expectation that they contain a higher fraction of massive stars. Instead no correlation is present for SNIa. The SN frequency does not correlate with the galaxy activity probably because searches are unable to discover $\mathrm{SNe}$ in the nuclear regions of galaxies.

A number of SNe with spectra similar to those of AGN exist. Their characteristic features are explained with explosion of $\mathrm{SNe}$ in dense environments, reminding of cSNR's invoked in the starburst model for AGNs. Some recent, peculiar SNe seem linked to GRB's opening the possibility that at least some GRB's arise from this kind of stellar explosion.
\end{abstract}

\section{Introduction}

The common property of all kinds of SNe, independent of the explosion mechanism, is the amount of energy (of order $10^{51} \mathrm{ergs}$ ) emitted as kinetic and luminous energy. After that each SN type has distinctive characteristics.

Type Ia SNe are discovered in all kind of galaxies and show a high degree of homogeneity. They have been explained by thermonuclear explosions of accreting WD's, hence associated with Population I. Recent findings have shown that some SNIa reached magnitudes at maximum considerably different from the average and challenged the standard scenario.

Type II and Ib/c SNe result instead from the explosions of massive stars $\left(\mathrm{M}>8 \mathrm{M}_{\odot}\right)$ via core collapse. The optical displays are governed mainly by the configurations of the precursor stars at the moment of explosion and by the amount of radioactive material. If the stars retain the $\mathrm{H}$ envelopes, the $\mathrm{SN}$ shows Balmer lines and is classified as type II. On the other side, if the $\mathrm{H}$ envelope (and, in cases, even the He layer) has been lost by the progenitor via wind or binary interaction, the SN is classified as SNIb (or SNIc). The discovery of few but significant cases of transitions from early SNII to late SNIb/c spectra, 
has firmly established a physical link between these classes of objects, hence confirmed the association of $\mathrm{SNIb} / \mathrm{c}$ with young progenitors.

For their descent from different progenitors, SNe have been used in the past as probes of stellar populations in galaxies. In particular, SNII and Ib/c have been used as indicators of star formation (e.g. Petrosian \& Turatto 1990, 1995). Clearly differently from other SFR indicators such as FIR luminosity, intensity of recombination lines, etc., $\mathrm{SNe}$ cannot be used to test individual galaxies but can give the SFR in a given sample.

In addition to these topics, we will discuss the properties of a particular subclass of type II SNe, the SNIIn, which for their overall spectral similarity to AGN are of special interest to the subject of this conference. Finally, the recent association of some SN to GRB's will be briefly addressed.

\section{The Supernova and SF rates}

Given a sample of $N_{\text {gal }}$ galaxies, the rate of $\mathrm{SNe}$ per unit time and luminosity is given by

$$
\frac{N_{S N}}{\sum_{1}^{N_{g a l}} t_{i} L_{i} k_{i}}
$$

where $N_{S N}$ is the number of $\mathrm{SNe}$ discovered in the sample, $t_{i}$ and $L_{i}$ are the surveillance time and the luminosity of the $i$-th galaxy, and $k_{i}$ is the correction term, relative to that galaxy, which takes into account different search biases.

Currently, the efforts to improve the accuracy of the observational rates are focused in increasing the statistics, i.e. $N_{S N}$, and in determining appropriate corrections for the selection effects (cfr. Cappellaro et al. 1997 and references therein).

By grouping the databases of five SN searches (updated versions of those described in Cappellaro et al. 1997) we have compiled a sample of about $\simeq 10^{4}$ RC3 galaxies in which $137 \mathrm{SNe}$ have been discovered, the largest SN sample ever used for such purposes. The results are summarized in Table 1 . Note that the rates scale as $(\mathrm{H} / 75)^{2}$. The reported errors take into account the statistics of $\mathrm{SNe}$ and the uncertainty on the input parameters and the selection effects.

Table 1. The rate of Supernovae in a sample of about $10^{4} \mathrm{RC} 3$ galaxies and $137 \mathrm{SNe}$.

\begin{tabular}{|c|c|c|c|c|c|c|c|}
\hline galaxy & & of S & & & rate & SNu] & \\
\hline type & Ta & $\mathrm{Ib}$ & II & $\mathrm{Ta}$ & $\mathrm{Ib}$ & II & AII \\
\hline E-SO & 22.0 & & & $0.18 \pm 0.06$ & $<0.01$ & $<0.02$ & $0.18 \pm 0.06$ \\
\hline SOa-Sb & 18.5 & 5.5 & 16.0 & $0.18 \pm 0.07$ & $0.11 \pm 0.06$ & $0.42 \pm 0.18$ & $0.72 \pm 0.14$ \\
\hline Scd-Sd & 22.4 & 7.2 & 31.5 & $0.21 \pm 0.08$ & $0.14 \pm 0.07$ & $0.86 \pm 0.36$ & $1.21 \pm 0.46$ \\
\hline Other & 6.8 & 2.2 & 5.0 & $0.40 \pm 0.19$ & $0.22 \pm 0.17$ & $0.65 \pm 0.37$ & $1.26 \pm 0.40$ \\
\hline All & 69.6 & 14.9 & 52.5 & $0.20 \pm 0.06$ & $0.08 \pm 0.04$ & $0.40 \pm 0.15$ & $0.68 \pm 0.11$ \\
\hline
\end{tabular}

Somewhat different from older determinations (e.g. Cappellaro et al. 1993) the rate of SNIa does not increase significantly from ellipticals to late spirals. This is due both to a new determination of the correction factors (Cappellaro 
et al. 1997) and to new galaxy data (here we adopted the RC3 updated version from CDS, 1995). Instead, the rate of core-collapse $\mathrm{SNe}$ is rapidly increasing from zero in ellipticals to about $0.5 \mathrm{SNu}$ in early spirals and close to $1 \mathrm{SNu}$ in late spirals. This resembles the average increase of SFR along the Hubble sequence (Kennicutt 1998).

The correlation of SN rates with other SFR indicators has been controversial. For instance, Kennicutt (1984) found that the rate of SNII was strongly correlated to the total massive star formation rate in galaxies as measured by their total $\mathrm{H}_{\alpha}$ emission. Instead, on a small sample of galaxies, Richter \& Rosa (1989) suggested that the star formation determined from the UV luminosities was only slightly correlated to the SN productivity. Statistical analyses of the star forming Markarian galaxies and of AGN host galaxies have not found enhanced SN rates but only a tendency of SNe to explode closer to the nuclear regions (Turatto et al. 1989; Petrosian \& Turatto 1990).

We have computed again the rates of SNe in AGN host galaxies by extracting from our general sample the active galaxies according to Veron-Cetty \& Veron (1998). Though only $3 \%$ of the galaxies of the sample are active, they hosted $12 \%$ of SNe. Yet, the rate expressed in $\mathrm{SNu}$ is not different from that in normal galaxies. This apparent contradiction is due to the fact that both the average control time and luminosity of the AGN of the sample are higher than normal galaxies. In other words, AGN host galaxies were surveyed better and they are bigger than other galaxies of our sample. A similar conclusion, i.e. that the SN frequency in star forming galaxies is not enhanced, was reached by Richmond et al. (1998) from a dedicated SN search in a sample of 142 starburst galaxies. We note however that $\mathrm{SN}$ rates are not able to monitor $\mathrm{SNe}$ in the central regions of the galaxies because of increased extinction.

The correlation of SN rate with SF indicators is possibly hidden by normalization factors. Actually, the fact that the rate of core-collapse $\mathrm{SNe}$ in $\mathrm{SNu}(\mathrm{B})$ increases towards late spirals indicates that the blue luminosity is not an optimal indicator of current SF (e.g. Sage and Solomon 1989), otherwise one should expect a constant rate per unit luminosity.

Alternatively one may use $\mathrm{L}_{F I R}$, which is often used as a direct measure of the SFR. Only $30 \%$ of our galaxy sample have FIR data. The results, reported in Tab. 2, show that the rate of core-collapse SNe normalized to FIR luminosity increases along the Hubble sequence. This means also that $\mathrm{L}_{F I R}$ is not a universal measure of SFR.

A possible explanation is that the FIR luminosity of the galaxies is the sum of two components, one taking place in the circumnuclear regions and one in the disks. The two components are decoupled with the circumnuclear component strongly correlated to the nuclear SF. Instead the disk component is contaminated by the IR emission of the dust heated by evolved stellar populations, which varies significantly with the Hubble type. On the other hand, as said before, because of strong extinction optical SN searches cannot discover objects in the nuclear regions and the observed SN rates test SFR only in the disks. The SN rates in units of FIR luminosity shows the same dependence as a function of the Hubble types as those expressed in $\mathrm{SNu}$ (B) (cfr. Tab. 2).

More reliable to this purpose can be the FIR excess, $\mathrm{L}_{F I R} / \mathrm{L}_{B}$, though also this SF indicator has been questioned in the past because both the FIR and B 
Table 2. The rate of Supernovae in units of FIR luminosity.

\begin{tabular}{|c|c|c|c|}
\hline galaxy & \multicolumn{3}{|c|}{ rate $\mid S N u_{F I R}$} \\
\hline type & Ia & $\mathrm{II}+\mathrm{Ib} / \mathrm{c}$ & All \\
\hline E-SO & $1.8 \pm 0.8$ & & $1.8 \pm 0.8$ \\
\hline SOa-Sb & $0.6 \pm 0.2$ & $2.0 \pm 0.5$ & $2.7 \pm 0.5$ \\
\hline Sbc-Sd & $0.6 \pm 0.1$ & $3.5 \pm 0.6$ & $4.1 \pm 0.6$ \\
\hline All & $0.7 \pm 0.1$ & $2.5 \pm 0.3$ & $3.2 \pm 0.3$ \\
\hline
\end{tabular}

luminosity somehow correlate with the SFR (e.g. Kennicutt 1998). In Tab. 3 the $\mathrm{SN}$ rates in $\mathrm{SNu}(\mathrm{B})$ for three groups of galaxies with different FIR excess, i.e. those with strong FIR excess, those with low excess and those with no FIR flux, are shown. A trend is evident for type $\mathrm{II}+\mathrm{Ib} / \mathrm{c} \mathrm{SNe}$ with higher rates in galaxies with stronger FIR excess. Such effect is not seen for SNIa. One may conclude that the FIR excess is a better SFR indicator than $\mathrm{L}_{F I R}$.

Table 3. $\mathrm{SN}$ rates in $\mathrm{SNu}(\mathrm{B})$ for galaxies with different FIR excess

\begin{tabular}{|c|c|c|c|c|c|c|}
\hline \multirow{2}{*}{$\begin{array}{l}\text { galaxy } \\
\text { type }\end{array}$} & \multicolumn{2}{|c|}{ not detected by IRAS } & \multicolumn{2}{|c|}{$L_{F I R} / L_{B}<0.35$} & \multicolumn{2}{|c|}{$L_{F I R} / L_{B}>0.35$} \\
\hline & la & $\mathrm{II}+\mathrm{Ib} / \mathrm{c}$ & la & $\mathrm{II}+\mathrm{Ib} / \mathrm{c}$ & & $\mathrm{II}+\mathrm{lb} / \mathrm{c}$ \\
\hline E-SO & $0.2 \pm 0.1$ & & $0.4 \pm 0.2$ & & $<0.5$ & \\
\hline $\mathrm{SOa-Sb}$ & $0.2 \pm 0.1$ & $0.3 \pm 0.2$ & $0.2 \pm 0.1$ & $0.5 \pm 0.2$ & $0.3 \pm 0.1$ & $1.1 \pm 0.4$ \\
\hline Scd-Sd & $0.3 \pm 0.1$ & 0.7 & $0.2 \pm 0.1$ & $1.0 \pm 0.2$ & $0.2 \pm 0.1$ & $1.2 \pm 0.3$ \\
\hline All & $0.2 \pm 0.1$ & $0.2 \pm 0.1$ & $0.2 \pm 0.1$ & $0.9 \pm 0.1$ & $0.3 \pm 0.1$ & $1.1 \pm 0.2$ \\
\hline
\end{tabular}

As a further check we have estimated the $\mathrm{SN}$ rates in galaxies with different colors extending the analysis carried out by Tammann (1974). An increase of the $\mathrm{SN}$ rate of type $\mathrm{II}$ and $\mathrm{Ib} / \mathrm{c} \mathrm{SNe}$ is visible in all galaxy bins while that of SNIa is insensitive to the colors of the host galaxies. This is expected since bluer galaxies are expected to host more young stars than red ones.

Table 4. SN rates for galaxies of different (B-V) colors

\begin{tabular}{lccc|ccc}
\hline galaxy & \multicolumn{3}{c|}{ rates $[\mathrm{SNu}]$} & \multicolumn{2}{c}{ rates $[\mathrm{SNu}]$} \\
type & $\left\langle(B-V)_{T}^{0}\right\rangle$ & $\mathrm{Ia}$ & $\mathrm{II}+\mathrm{Ib}$ & $\left\langle(B-V)_{T}^{0}\right\rangle$ & $\mathrm{Ia}$ & $\mathrm{II}+\mathrm{Ib}$ \\
\hline E-S0 & 0.86 & $0.3 \pm 0.1$ & & 0.95 & $0.2 \pm 0.1$ & \\
S0a-Sb & 0.60 & $0.2 \pm 0.1$ & $0.6 \pm 0.2$ & 0.80 & $0.2 \pm 0.1$ & $0.5 \pm 0.2$ \\
Sbc-Sd & 0.45 & $0.1 \pm 0.1$ & $1.5 \pm 0.3$ & 0.62 & $0.3 \pm 0.1$ & $0.9 \pm 0.2$ \\
All & 0.56 & $0.2 \pm 0.1$ & $1.0 \pm 0.2$ & 0.88 & $0.2 \pm 0.1$ & $0.1 \pm 0.1$ \\
\hline \multicolumn{7}{r}{ in SNu(B) }
\end{tabular}

We conclude, therefore, that the rates of core-collapse SNe correlate with the FIR excess and the galaxy colors once the dependence on the Hubble type is removed. This finding confirms that the SN rates can be used as probes of SF. Conversely, Tables 3 and 4 confirm that the parent population of type II and $\mathrm{Ib} / \mathrm{c} \mathrm{SNe}$ is constituted by young massive stars. 


\subsection{Supernovae and galaxy activity}

The source of the energy in active galaxies is, according to the standard model, the accretion of material onto a supermassive nuclear black hole. As an alternative, Terlevich and collaborators have developed in the last decades the starburst model, which does not require the presence of a black hole to explain the AGN's observed properties (Terlevich et al. 1992, 1995). The model explain the time variability, X-ray and radio emission, BLR properties and NLR ionization by means of compact Supernova Remnants (cSNR), i.e. ordinary SNRs evolving in a dense $\left(10^{6} \mathrm{~cm}^{-3}\right)$ circumstellar medium which are able to radiate most of their kinetic energy in a few years.

Without entering the dispute of how good the starburst model is in explaining the AGN properties as compared with the standard model, there is no doubt that a number of SNe with spectra impressively similar to those of AGN exist. Such Seyfert 1 impostors, as named by Filippenko (1989), or SNIIn, as most commonly called by supernovists, are indeed normal $\mathrm{SNe}$ exploding in dense environments.

The best example of such a SNe is 1988Z (Stathakis \& Sadler 1991, Turatto et al. 1993). Contrary to normal SNII, the spectrum of SN $1988 \mathrm{Z}$ does not show the P-Cygni profiles but only relatively narrow emission with complex profiles. Shortly after the explosion the most intense component of $\mathrm{H} \alpha$ was $2200 \mathrm{~km} \mathrm{~s}^{-1}$ wide (FWHM) and less intense, but clearly visible, were a broad component (FWHM $15000 \mathrm{~km} \mathrm{~s}^{-1}$ ) and an unresolved one (FWHM $<700 \mathrm{~km}$ $\mathrm{s}^{-1}$ ). A number of unresolved faint lines of very high ionization (e.g. [FeX], [FeVII], [FeVI]) were also present. The line intensity ratios of SN $1988 \mathrm{Z}$ indicate densities of the order of $10^{6}$ to $10^{7} \mathrm{~cm}^{-3}$ and this was confirmed by the nondetection of the [NII] 6548-6583A lines.

In addition to the peculiar spectral features, the temporal evolution of this object was unusually slow. After the maximum at about $\mathrm{M}=-18$ the $\mathrm{SN}$ declined only $3 \mathrm{mag}$ in $3 \mathrm{yr}$ in the R band while normal SNe fade the same amount in few months. Indeed, despite the relatively large distance, SN $1988 \mathrm{Z}$ is still reachable nowadays, 10 years after the discovery.

Several supernovae with narrow emission lines have been grouped in the SNIIn subclass (Schlegel 1990). Though it is not yet clear if all such objects undergo similar physical processes, it is clear that at least a fraction of them share also other features of SN 1988Z, i.e. relatively narrow lines, very slow evolution, radio and X-ray emission and in some cases, weak unresolved lines of very high ionization.

Remarkable is the spectral similarity of SN $1988 \mathrm{Z}$ with SN 1995N (Turatto et al. 1998) and, to a lesser extent, with SN 1995G (Pastorello 1998). Figure 1 compares the spectra of these three SNe to that of a Sy 1.5 galaxy.

The observations of these SNe can be interpreted by a model in which the bulk of emission is due to the interaction of the ejecta with a dense CSM (Chugai $\&$ Danziger 1994). The broad emissions arise at the contact discontinuity between the high-velocity shocked ejecta and the shocked circumstellar wind, while the lines of intermediate widths come from shocked clumps embedded in the rarefied medium. The model also explains well the radio and X-ray emission from the shocked material and the narrow coronal lines, since temperatures as high as $10^{6} \mathrm{~K}$ are reached. 


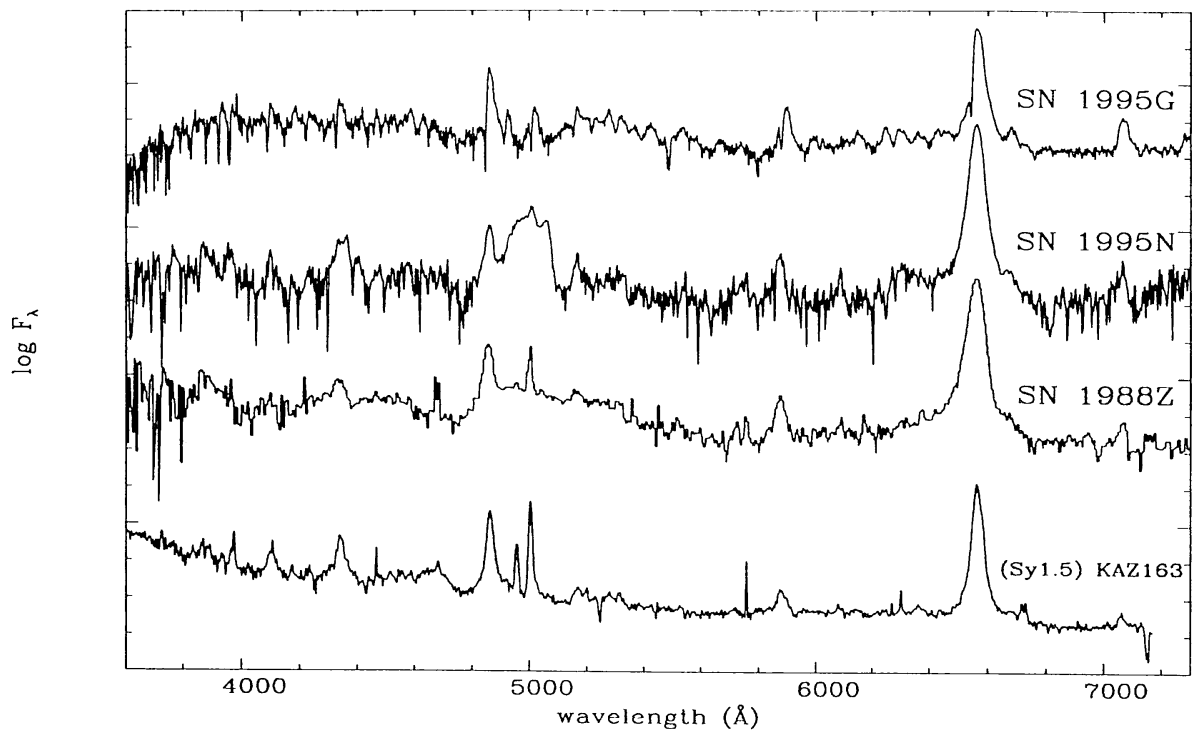

Figure 1. Comparison of the spectra of three SNIIn, SNe 1988Z, $1995 \mathrm{G}$ and $1995 \mathrm{~N}$ at about 2 years past maximum, with a Seyfert 1.5 spectrum. 
The existence of SNe with spectra similar to AGN, which are interpreted by the interaction of the ejecta with dense circumstellar matter, renews interest in the starburst model.

\section{3. $\gamma$-ray activity}

Gamma Ray Bursts are one of the mysteries of current astronomy. A recent breakthrough was obtained thanks to the Italian-Dutch Beppo-SAX satellite which allowed the optical identifications of a number of GRB's. It turned out that some of them are related to some sort of activity in distant galaxies. One outstanding exception was GRB 980425 associated with the relatively nearby $(\mathrm{z}=0.0085) \mathrm{SN} 1998 \mathrm{bw}$. This $\mathrm{SN}$ was discovered within the GRB error box soon after the detection (Galama et al. 1998). The a posterior probability to find a SN inside the error box of WFC is only $10^{-4}$

The spectrum appeared unusual while showing very broad features typical of SNe. The light curve was also unprecedented. Extensive multifrequency observations, still in progress, are showing that this is the most powerful radio $\mathrm{SN}$ ever, and that relativistic outflow is required to explain the spectral features. If the SN and the GRB are indeed associated, i.e. if we place the GRB at the distance of the SN parent galaxy, the peak luminosity and total budget of the $\gamma$-ray event are orders of magnitude fainter than other GRB's.

The properties of SN 1998bw have been modeled by the collapse into a black hole of a $\mathrm{C}+\mathrm{O}$ star with mass of $12-15 \mathrm{M}_{\odot}$. A core like this might originate from a progenitor with initial mass of about $40 \mathrm{M}_{\odot}$ stripped of its $\mathrm{H}$ and He either via stellar wind or binary interaction. The explosion energy required is huge for a $\mathrm{SN}$, of the order of $10^{52} \mathrm{ergs}$, and the mass of ${ }^{56} \mathrm{Ni}$ produced is $0.6-0.8 \mathrm{M}_{\odot}$ (Iwamoto et al. 1998). High energy photons are emitted via the synchrotron mechanism when, after the shock breakout, external layers are highly accelerated to produce a relativistic shock with the circumstellar material. It must be noted however that, although this was a relatively weak GRB, the $\gamma$-rays predicted by such a model are not sufficient to explain the observations unless the emission was collimated (Iwamoto et al. 1998).

The discovery of this SN-GRB event has triggered the search for angular and temporal correlations of other SNe with detected GRB's. The results are contradictory (Wang \& Wheeler 1998, Kippen et al. 1998). The straight association of normal SNe with GRB's seems extremely weak, while it seems possible that faint GRB's are associated with peculiar SNe.

Woosley et al. (1998) have pointed out among others the interesting association of SN 1997cy with GRB 970514, which are compatible both in epoch and location. This SN has been extensively observed by us at ESO and the preliminary reduction of the data shows that both the spectra and the light curve share several of the characteristics of SN 1988Z, namely narrow emission lines with broad wings (Fig. 2) and the slow light curve. The study of this SN is at present in progress but there is evidence that such event was also particularly energetic: with $\mathrm{M}_{V}(\max )>-20$ this would be the brightest $\mathrm{SN}$ ever.

The discovery and the study of cases like these will finally prove the association of GRB's with peculiar SNe and define the role of SNe in powering these extraordinary events. 


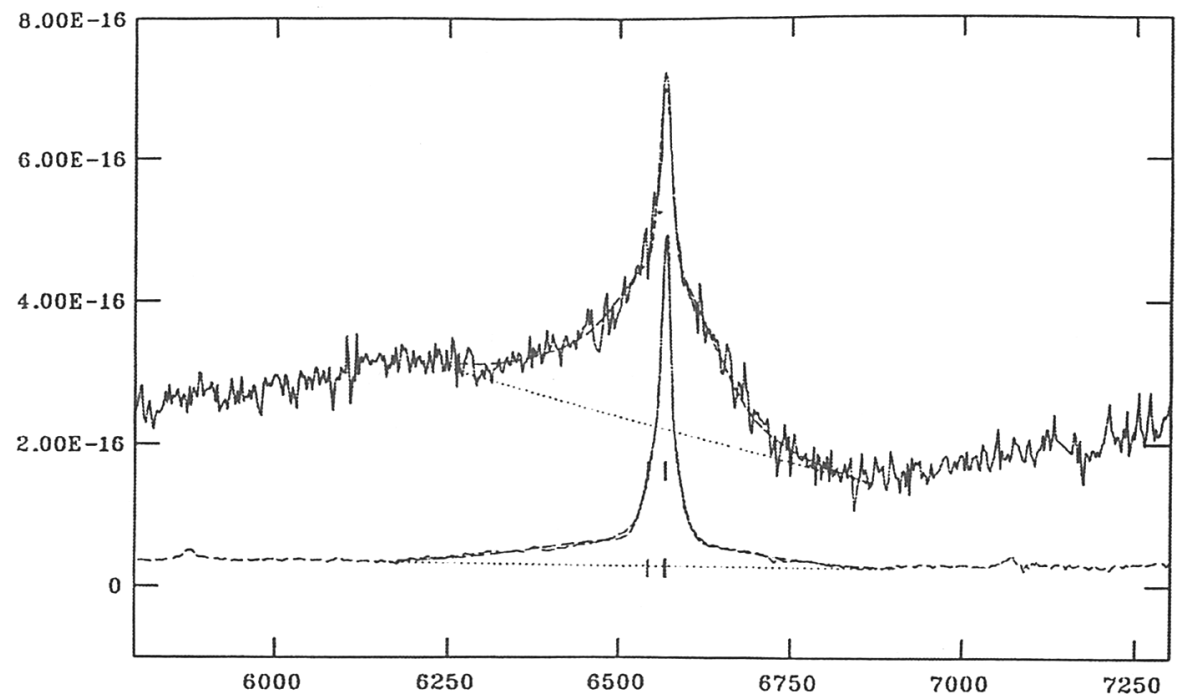

Figure 2. Comparison of the $\mathrm{H}_{\alpha}$ emission of $\mathrm{SNe} 1988 \mathrm{Z}$ (bottom) and 1997cy (top) in spectra taken short after maximum. Relatively narrow lines with broad wings are evident in both cases. 
Acknowledgments. We are grateful to Andrea Pastorello for the preparation of the figures.

\section{References}

Cappellaro, E. Turatto, Benetti, S., M. Tsvetkov, D.Yu. Bartunov, Makarova, I.N., 1993, A\&A, 273, 383

Cappellaro, E. Turatto, M. Tsvetkov, D.Yu. Bartunov, O.S. Pollas, C. Evans, R. Hamuy, M., 1997, A\&A, 322, 431

Chugai, N., Danziger, I.J., 1994, MNRAS, 268, 173

Filippenko, A., 1989, AJ, 97, 726

Galama, T. J. Vreeswijk, P. M. Van Paradijs,et al., 1998, Nature, 395, 670

Iwamoto, K. Mazzali, P. A. Nomoto, et al., 1998, Nature, 395, 672

Kennicutt, R.C., 1984, AJ, 277, 361

Kennicutt, R.C., 1998, preprint astro-ph 9807187

Kippen, R. M. Briggs, M. S. Kommers, J.M., et al., 1998, ApJ, 506, L27

Pastrorello, A., 1998, Tesi di laurea, Univarsità di Padova

Petrosian, A.R., Turatto, M., 1990, A\&A239, 63

Petrosian, A.R., Turatto, M., 1995, A\&A297, 49

Richmond, M.W., Filippenko, A., Galisky, J., 1998, PASP, 110, 553

Sage, L.J., Solomon, P.M., 1989, ApJ, 344, 204

Stathakis, R.A., Sadler, E.M., 1991, MNRAS, 250, 786

Tammann, G.A., 1974, Supernova and Supernova Remnants, ed. C.B. Cosmovici, Dordrecht, Reidel, p.155

Terlevich, R.J., Tenorio-Tagle, G., Franco, J., Melnick, J., 1992, MNRAS, 255, 713

Terlevich, R.J., Tenorio-Tagle, G., Franco, J., Rozyczka, M., Melnick, J., 1995, MNRAS, 272, 192

Turatto, M., Cappellaro, E. Danziger, I. J. Benetti, S. Gouiffes, C. Della Valle, M., 1993, MNRAS, 262, 128

Turatto, M., Benetti, S., Cappellaro, E., Danziger, I. J. Mazzali, P., 1998, SN 1987A: ten Years after, eds. Phillips, M.M., Suntzeff, N.B., Fifth $\mathrm{ESO} / \mathrm{CTIO} / \mathrm{LCO}$ Workshop, in press

Veron-Cetty, M.P., Veron, P., 1998, Catalogue of Quasars and Active Galaxy Nuclei, ESO Scientific Report 18.

Woosley, S.E., Eastman, R.G., Schmidt, B.P., 1998, ApJ, in press 